\title{
A workflow for patient-specific fluid-structure interaction analysis of the mitral valve: A proof of concept on a mitral regurgitation case
}

\author{
Benedetta Biffica, ${ }^{\mathrm{a}, *}$, Maurizio Gritti ${ }^{\mathrm{c}}$, Agata Grasso ${ }^{\mathrm{d}}$, Elena G. Milano ${ }^{\mathrm{a}}$, Marianna Fontana , \\ Hamad Alkareef ${ }^{a}$, Joseph Davar ${ }^{\mathrm{d}}$, Paramijit Jeetley ${ }^{\mathrm{d}}$, Carol Whelan ${ }^{\mathrm{d}}$, Sarah Anderson ${ }^{\mathrm{c}}$, \\ Donatella Lorusso ${ }^{c}$, Emilie Sauvage ${ }^{a}$, Giorgia Maria Bosi ${ }^{a}$, Silvia Schievano ${ }^{a}$, \\ Claudio Capelli ${ }^{\mathrm{a}}$ \\ ${ }^{a}$ Centre for Clinical Cardiovascular Engineering, UCL Institute of Cardiovascular Science \& Great Ormond Street Hospital for Children, London, UK \\ ${ }^{\mathrm{b}}$ Department of Medical Physics and Biomedical Engineering, University College London, London, UK \\ ' The National Amyloidosis Centre, Division of Medicine, UCL Medical School, Royal Free Hospital, London, UK \\ d Department of Cardiology, Royal Free Hospital, London, UK
}

\section{A R T I C L E I N F O}

\section{Article history:}

Received 28 January 2019

Revised 24 July 2019

Accepted 29 September 2019

Available online $\mathrm{xxx}$

\section{Keywords:}

Cardiac magnetic resonance

3D transoesophageal-echocardiogram

Patient-specific modelling

Left heart

Mitral valve

Fluid-structure interaction simulations

Smoothed particles hydrodynamics

\begin{abstract}
A B S T R A C T
The mechanics of the mitral valve (MV) are the result of the interaction of different anatomical structures complexly arranged within the left heart (LH), with the blood flow. MV structure abnormalities might cause valve regurgitation which in turn can lead to heart failure. Patient-specific computational models of the MV could provide a personalised understanding of MV mechanics, dysfunctions and possible interventions. In this study, we propose a semi-automatic pipeline for MV modelling based on the integration of state-of-the-art medical imaging, i.e. cardiac magnetic resonance (CMR) and 3D transoesophagealechocardiogram (TOE) with fluid-structure interaction (FSI) simulations. An FSI model of a patient with MV regurgitation was implemented using the finite element (FE) method and smoothed particle hydrodynamics (SPH). Our study showed the feasibility of combining image information and computer simulations to reproduce patient-specific MV mechanics as seen on medical images, and the potential for efficient in-silico studies of MV disease, personalised treatments and device design.
\end{abstract}

(C) 2019 The Authors. Published by Elsevier Ltd on behalf of IPEM.

This is an open access article under the CC BY license. (http://creativecommons.org/licenses/by/4.0/)

\section{Introduction}

The mechanics and function of the mitral valve (MV) depend on the interaction of different anatomical structures - annulus, leaflets, chordae tendinae and papillary muscles (PMs) - complexly arranged within the left heart (LH), with the blood flow. Structurally, the two MV leaflets are attached to the valve annulus, at the junction between the left atrium (LA) and left ventricle (LV) [1]. The role of the MV is to open during diastole and close in systole, thus allowing unidirectional flow from the LA into the LV. Structural abnormalities of the MV or functional abnormalities of

List of Abbreviations:: AoV, Aortic valve; CMR, Cardiac magnetic resonance; EDV, End-diastolic volume; ESV, End-systolic volume; FE, Finite element; FSI, Fluidstructure interaction; LA, Left atrium; LH, Left heart; LV, Left ventricle; LVOT, Left ventricular outflow tract; MR, Mitral regurgitation; MV, Mitral valve; MVR, Mitral vortex ring; PMs, Papillary muscles; SPH, Smoothed particle hydrodynamics; SV, Stroke volume; TOE, Transoesophageal echocardiography; WH, Whole heart.

Corresponding author at: Centre for Clinical Cardiovascular Engineering, Department of Children's Cardiovascular Disease, UCL Institute of Cardiovascular Science \& Great Ormond Street Hospital for Children, London, UK

E-mail address: b.biffi@ucl.ac.uk (B. Biffi). the LV might result in abnormal leaflet closure during systole and regurgitation of blood back into the LA, which in turn leads to chronic LV volume overload and possibly evolving into heart failure over time [2].

Open-heart surgery MV repair or replacement remains the standard of care for these cases [3], although, in the past decade, considerable efforts have been devoted to the development of less invasive (i.e. transcatheter) techniques [4-7], with several devices currently under development. Widespread use of such devices as seen for the aortic valve has not yet been achieved due to the complexity of the MV architecture, which makes it difficult to approach via catheter. In addition, MV disease is characterised by a wide spectrum of aetiologies, thus complicating the diagnosis and requiring an individualised approach in treatment selection (repair or replacement, interventional or surgical) $[4,8]$. In this context, computational models could offer a thorough evaluation of structural and hemodynamics factors of disease and potentially predict the outcomes of each procedure, thus providing a desirable preprocedural planning tool to enhance safety and efficacy [9-12].

Indeed, fluid-structural interaction (FSI) models which take into account the complex MV structure and function have been 
proposed over the last decade. Mesh-based FSI methods - the fluid mesh is fixed and the flow/structure interface is indirectly defined by altering the equilibrium equations [13] - based on the Arbitrary-Lagrangian-Eulerian formulation were first introduced by Kunzelman et al. [14] on an idealised MV and further tested by Lau et al. [15] on an anatomically sized MV in an idealised LV geometry to simulate MV edge-to-edge repair [16]. A patient-specific MV model was first reported by Ma et al. [17] with the immersed boundary method [18] implemented to simulate MV function with physiological, dynamic transvalvular pressure loads. The model has been extended in subsequent works from the same group by including a transversely isotropic material constitutive model for the MV tissue [19], and a patient-specific LV with an active and passive material model [20-21].

The main limitation of such mesh-based FSI methods $[22,23]$ is the lack of complete valve coaptation at systole. To overcome this, mesh-free methods such as smoothed particle hydrodynamics (SPH) have recently been proposed that allow simulation of complex physical problems, involving large deformation, fluid flow and FSI [24]. A MV FSI model achieved by using SPH and finite element (FE) was first validated by Toma et al. $[25,26]$ against an in-vitro setup and used to simulate the impact on regurgitation of different chordal ruptures. SPH-FE was also adopted by Mao et al. [27] to investigate both the aortic valve and MV structural responses and the LV hemodynamics in a realistic LH model during the entire cardiac cycle.

In this study, we present a workflow to set up a patient-specific FE-SPH model built by combining in vivo 3D anatomical patient data from different image modalities, and that can provide detailed information on both LH and the MV structures with personalised boundary conditions.

\section{Materials and methods}

A workflow was set up in this study to derive a patient-specific model from multi-modality medical images and to simulate pathophysiological conditions (Fig. 1). First, a fully automatic process was developed to extract the MV patient-specific virtual anatomical model by combining 3D anatomical information from cardiac magnetic resonance (CMR) imaging for the LH with 3D transoesophageal echocardiography (TOE) data for the MV. Then, such reconstructed patient-specific anatomical model, including
LH temporal information, was used to reproduce personalised MV mechanics and dysfunction.

\subsection{Image data acquisition and processing}

A 63-years-old male patient referred to our Centre for mitral regurgitation (MR) assessment underwent routine TOE and consecutive CMR investigation as part of the research protocol. Ethical approval was obtained for this study (London Central Research Ethics Committee) and the patient provided informed consent. CMR images were acquired on a 1.5-T Magnetom Aera (Siemens Medical Solutions, Erlangen, Germany) including the following two sets of sequences: (i) balanced steady-state free precession (bSSFP) 3D whole heart (WH) (isotropic voxel size of $1.4 \mathrm{~mm}$ ), acquired during the diastasis in free breathing and with ECG-and respiratory-gating; and (ii) retrospectively gated bSSFP cine images (CINE), acquired in breath-hold ( 24 frames per cardiac cycle) in the short-axis (SAX) from the valves plane to the apex (slice spacing $\sim 8 \mathrm{~mm}$, in-plane isotropic voxel size of $1.4 \mathrm{~mm}$ ). CMR clinical report provided information about $\mathrm{LV}$ volumes $(E D V=307 \mathrm{ml}$ and $\mathrm{ESV}=132 \mathrm{ml}$ ) and $\mathrm{MV}$ regurgitant volume. The latter, computed as the difference between left and right ventricle SVs, was recorded between 60 and $70 \mathrm{ml}$. The patient heart rate at the time of CMR was $75 \mathrm{bpm}$. TOE was performed using an iE33 xMatrix DS (Philips, Eindhoven, Netherlands) ultrasound machine with 3D zoomed MV leaflets and annulus acquisition for a full cardiac cycle (10 frames/cardiac cycle, isotropic voxel size of $0.4 \mathrm{~mm}$ ). Findings were of severe MR with one eccentric, anteriorly directed regurgitant jet due to posterior MV leaflet prolapse secondary to chordae rupture.

\subsubsection{CMR image processing}

To overcome the limitations of out of plane and axis error of CINE SAX images acquired at different breath holds, the SAX dataset was combined with the static WH $3 \mathrm{D}$ volume in order to create the LH 4D information. CINE SAX images at the same phase of the cardiac cycle were first stacked using DICOM slice spacing and orientation, thus providing a non-isotropic 3D dataset of the LH for each frame of the cardiac cycle. Synthetic WH images were obtained for each of the 24 acquisition frames via nonrigid image registration $[28,29]$ of the end-diastolic WH with each non-isotropic CINE stack. An atlas-based segmentation method

\section{Clinical data Fully automatic}

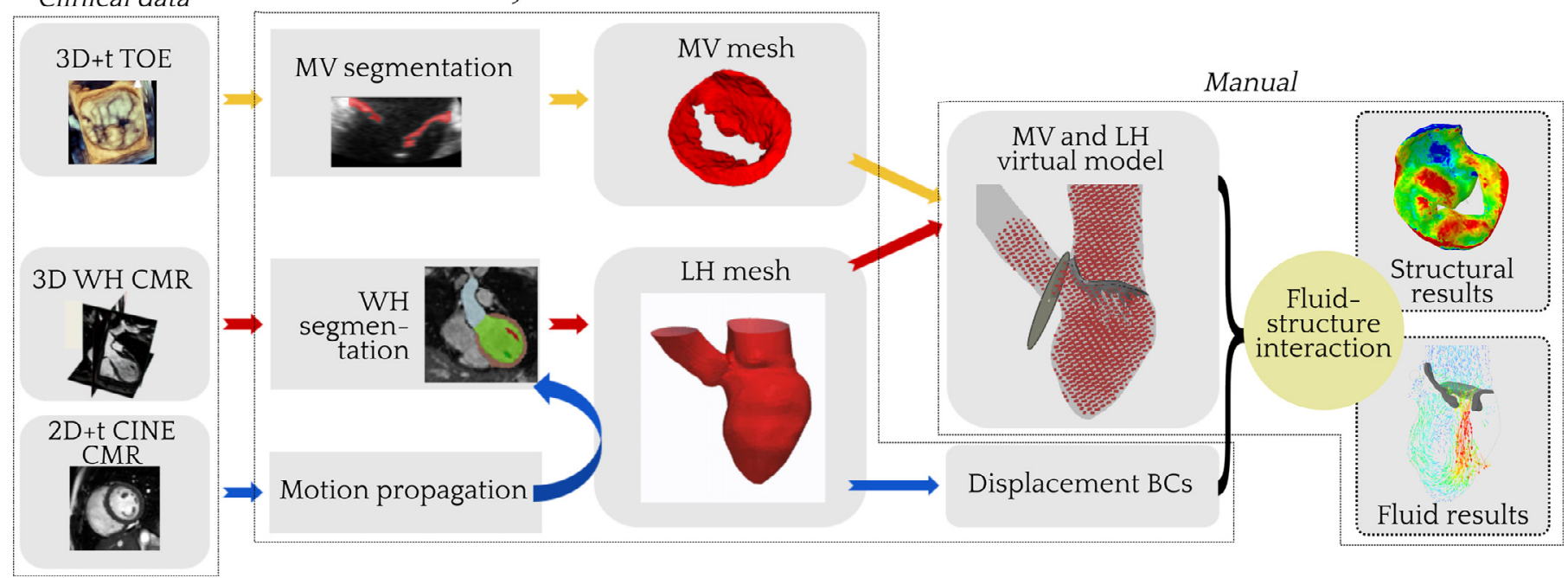

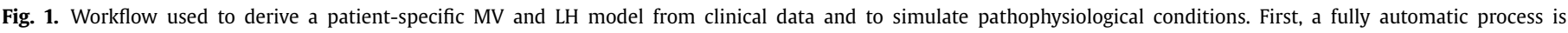

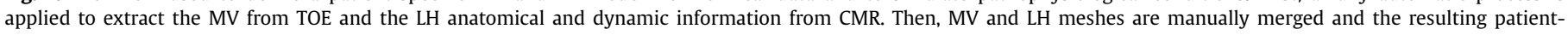
specific anatomical model, including LH temporal information, is used to reproduce personalised MV mechanics and dysfunction. 

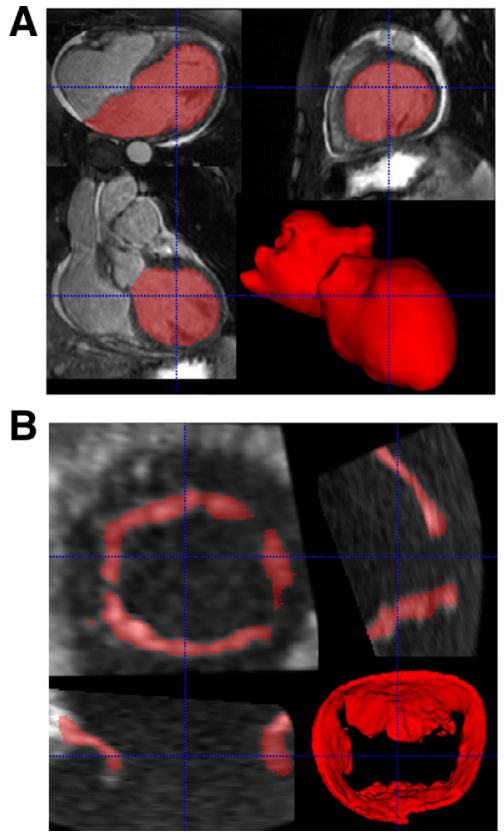

\section{C}

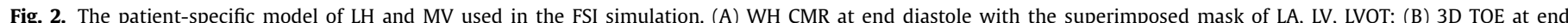

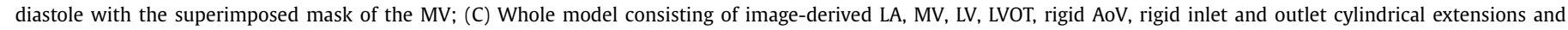

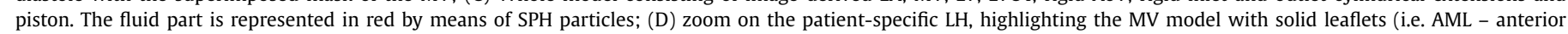

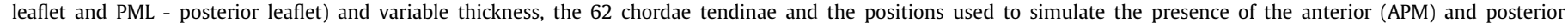
(PPM) PMs; (E) side and (F) top views of the MV model.

[30] able to label automatically the main cardiac structures was used to segment each of the synthetic WH images. An LH mask, produced by merging LA, LV endocardium and left ventricular outflow tract (LVOT), was obtained per each time step and converted into a surface mesh. The open source code Deformetrica [31] was used to non-rigidly register each LH surface mesh to the enddiastolic $\mathrm{LH}$, and the transformation was propagated to the latter thus achieving nodal correspondences between LH meshes at different time frames. The LH displacement field throughout the cardiac cycle was computed from nodal displacements and ultimately interpolated using piece-wise cubic splines. A detailed explanation of the method is reported in an earlier study by Biffi et al. [32].

\subsubsection{TOE image processing}

The automatic segmentation tool adopted for the CMR images was adapted to segment also MV leaflets and annulus from the 3D TOE images in the 10 frames during the cardiac cycle acquired for this patient. The segmentation included MV leaflets local thickness. The obtained end-diastolic MV segmentation was converted as a solid mesh and integrated into the end-diastolic LH model, while the MV meshes corresponding to the remaining frames were used for simulation validation purposes.

\subsection{Fluid-structure modelling approach: FE-SPH}

FE and SPH [24] methods were used within Abaqus/Explicit 2017 (SIMULIA, Dassault Systèmes) to implement the patientspecific FSI model of the LH and MV. After sensitivity analysis, the MV leaflets were meshed with 56,540 tetrahedral elements (average element edge length $=3 \mathrm{~mm}$ ) and modelled with uniform isotropic linear elastic material (density $=1146 \mathrm{~kg} / \mathrm{m}^{3}, E=3 \mathrm{MPa}$, Poisson ratio $=0.49$ ). As TOE image quality did not allow to identify the chordae tendinae insertion point on the patient image, the distribution of the chordae tendinae was adapted from adapted from the work of Baillargeon and co-workers [33,34] to interface with the patient-specific leaflets geometry and PMs position as seen on TOE and CMR images. Each chord was discretised using truss elements. The blood was modelled as incompressible Newtonian fluid (density $=1060 \mathrm{~kg} / \mathrm{m}^{3}$, dynamic viscosity $=0.004 \mathrm{~Pa} \mathrm{~s}$, $c_{0}=150 \mathrm{~m} / \mathrm{s}$ ) and discretised with 8749 uniformly distributed SPH particles (average particle distance at the beginning of the simulation was $3 \mathrm{~mm}$ ). Two rigid conduits were introduced to extend the LH model beyond LA and LVOT, thus providing a volume reservoir for SPH particles at inlet and outlet. The modified LH model was meshed with 19,456 triangular surface elements (average element edge length $=3 \mathrm{~mm}$ ). A simplified aortic valve was modelled with 2 semicircular rigid leaflets. The complete model is illustrated in Fig. 2.

The image-derived nodal displacements of the LH were imposed to LV, LA and part of the LVOT through a user-defined subroutine (VDISP). The flow rate through the MV, computed as the time derivative of the LV volume, was prescribed at the inlet by imposing corresponding translational velocity to a rigid disk acting as a piston. The disk was positioned $250 \mathrm{~mm}$ upstream the MV plane to allow enough fluid reservoir and avoid flow boundary effects. Outlet boundary conditions were reproduced by forcing opening and closing of the rigid aortic valve at the patient-specific cardiac cycle timings, and the systemic effect was mimicked by the presence of a reservoir of particles downstream the AoV. The effect of the papillary muscles was reproduced by a kinematic coupling between LV wall and extremities of the chordae tendinae. Using the implemented ghost particle method [35] a no-slip boundary condition was adopted between SPH particles and FE surfaces, and the FE-SPH interaction was activated via general contact algorithm. Starting from the end-diastolic configuration, two cardiac cycles were simulated and the second was considered for processing the results. The cardiac cycle duration was set at $0.8 \mathrm{~s}$ matching the patient heart rate. The structural parameters MV kinematics, leaflets stress and strain, and the fluid-dynamics parameters LV volume variations, global and local blood velocity were evaluated in the simulation. The blood velocity at the level of the MV tip was measured by averaging the velocity of the 


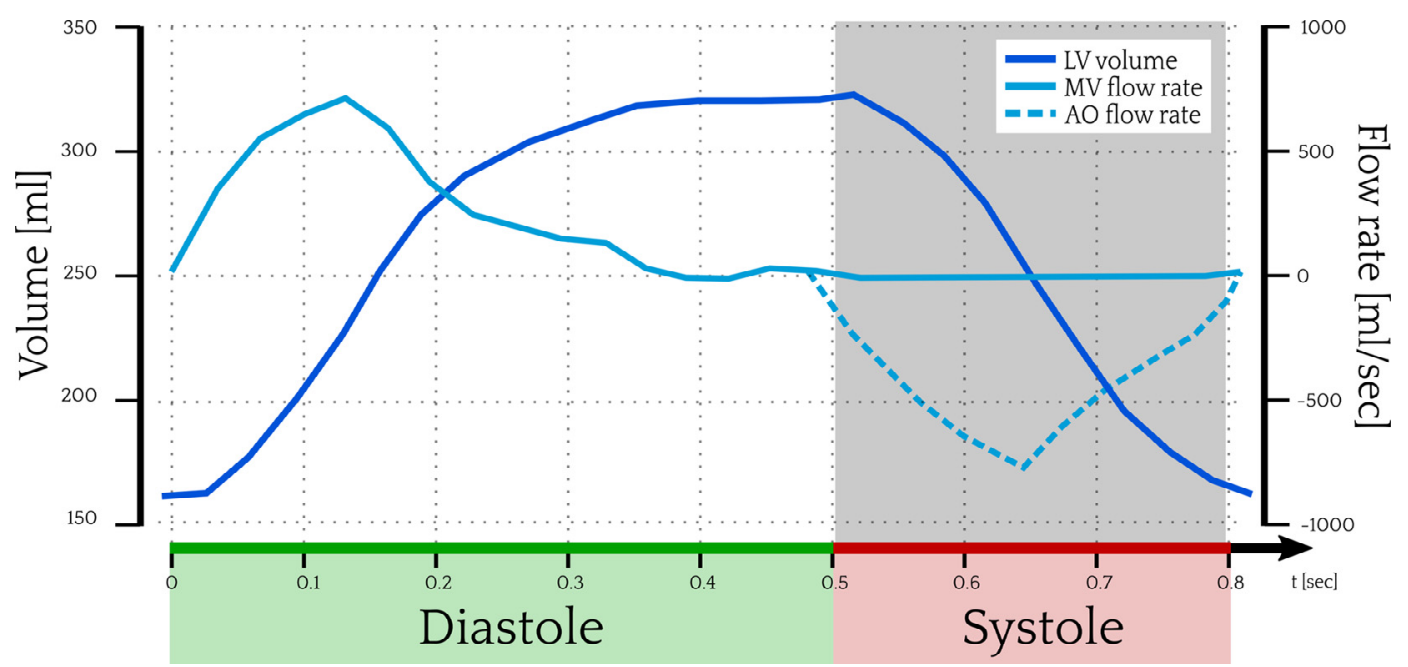

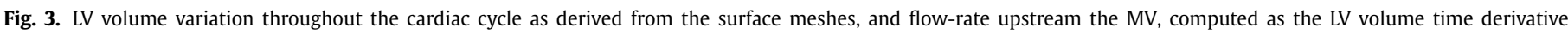
and imposed as the inlet boundary condition.

particles in a $10 \mathrm{~mm}$ diameter sphere positioned just below the open configuration of the MV tips. In addition, the time-dependent flow ejected in the aorta was computed by multiplying the average velocity normal to the aortic valve plane with the section area.

\section{Results}

The implemented image processing methods were able to successfully segment CMR and TOE images in a fully automatic way. Minimal user intervention was required to visually check the quality of the segmentation, and little to none manual refinement was applied. The time required to complete the model was $\sim 8 \mathrm{~h}$, i.e. $\sim 4 \mathrm{~h}$ for TOE and CMR processing providing MV and LH mesh on a double processor $2.93 \mathrm{GHz}$, RAM $48 \mathrm{~GB}$ machine, and $\sim 4 \mathrm{~h}$ for manual model setup including merging of MV and LH meshes, chordae tendinae insertion, boundary conditions and material properties definition. The SPH simulation required $\sim 48$ h to complete 2 cardiac cycles on a double processor $2.93 \mathrm{GHz}$, RAM 48GB machine.

LV mesh volumes varied from $322 \mathrm{ml}$ at end diastole to $163 \mathrm{ml}$ at end systole, resulting in $23.5 \%$ and $5.6 \%$ of discrepancy with the corresponding values computed from the CINE CMR. Fig. 3 shows the LV volume variation throughout the cardiac cycle as computed from CMR-derived surface meshes (dark blue), and its time derivative, i.e. the flow rate imposed at the inlet (cyan). The peak flow rate at diastole was found to be $800 \mathrm{ml} / \mathrm{s}$. All the results in the coming section will refer to the second simulated cardiac cycle.

The imposed image-derived displacement boundary conditions on the LV FSI model are superimposed to the 3-chamber long-axis reformatted CMR cine-images in Fig. 4 to illustrate the matching between the simulated and the CMR LV displacement at representative times of the cardiac cycle.

Realistic LH and MV fluid and structural behaviours were achieved throughout a full cardiac cycle by means of the FE-SPH interaction and thanks to image-derived patient-specific geometry and boundary conditions.

The MV kinematics resulting from successful interaction between simulated blood flow and MV structure was visually compared to the 3D MV TOE reconstructions at representative times of the cardiac cycle (Fig. 5). Despite an overall satisfactory similarity of 3D geometry in terms of MV leaflets aperture angle (side view) and valve orifice area (top view), a consistent delay in the simulated valve opening and closing times was recorded. Specifically, the simulated valve opening was $8 \mathrm{~ms}$ late compared to the TOE images $\left(t_{\text {sim,open }}=0.16 \mathrm{~s}\right.$ vs $\left.t_{\mathrm{TOE}, \mathrm{open}}=0.08 \mathrm{~s}\right)$, while the valve closure was $16 \mathrm{~ms}$ late $\left(t_{\text {sim,close }}=0.64 \mathrm{~s}\right.$ vs $\left.t_{\text {TOE, close }}=0.48 \mathrm{~s}\right)$.

Fig. 6 shows the MV leaflet maximum principal strain $(A-C)$ and maximum principal stress (D-F) distributions at representative opening/closing times from LV and LA views. Highest circumferential strains (41\%) were observed at the MV edge during sys-
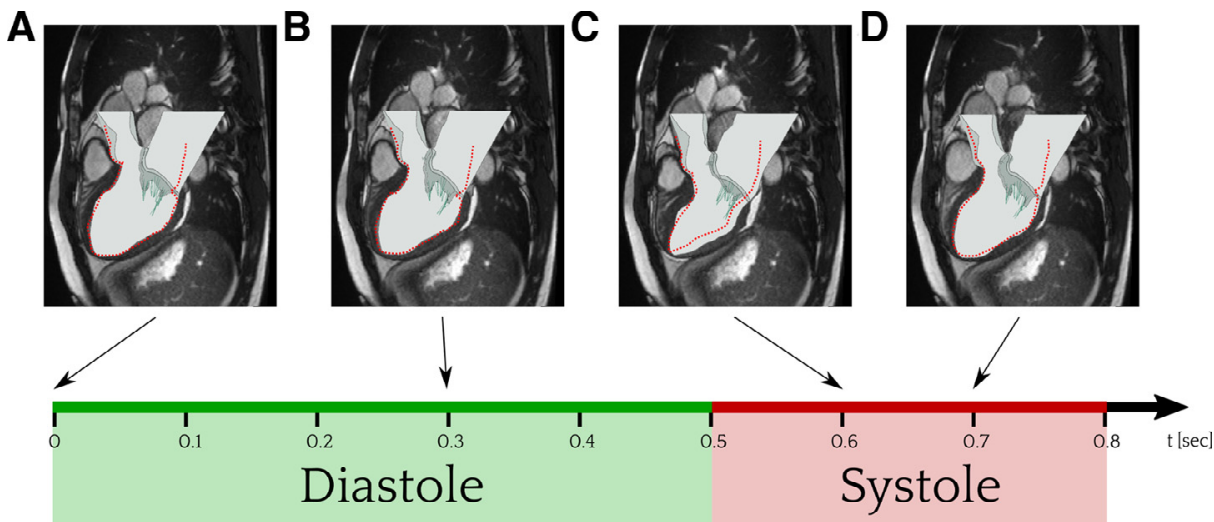

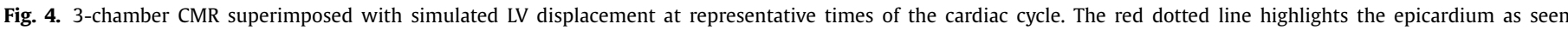
from CMR images. The difference seen at peak systole $(C)$ is due to the presence of the PMs which have been included in the LV blood pool during segmentation. 


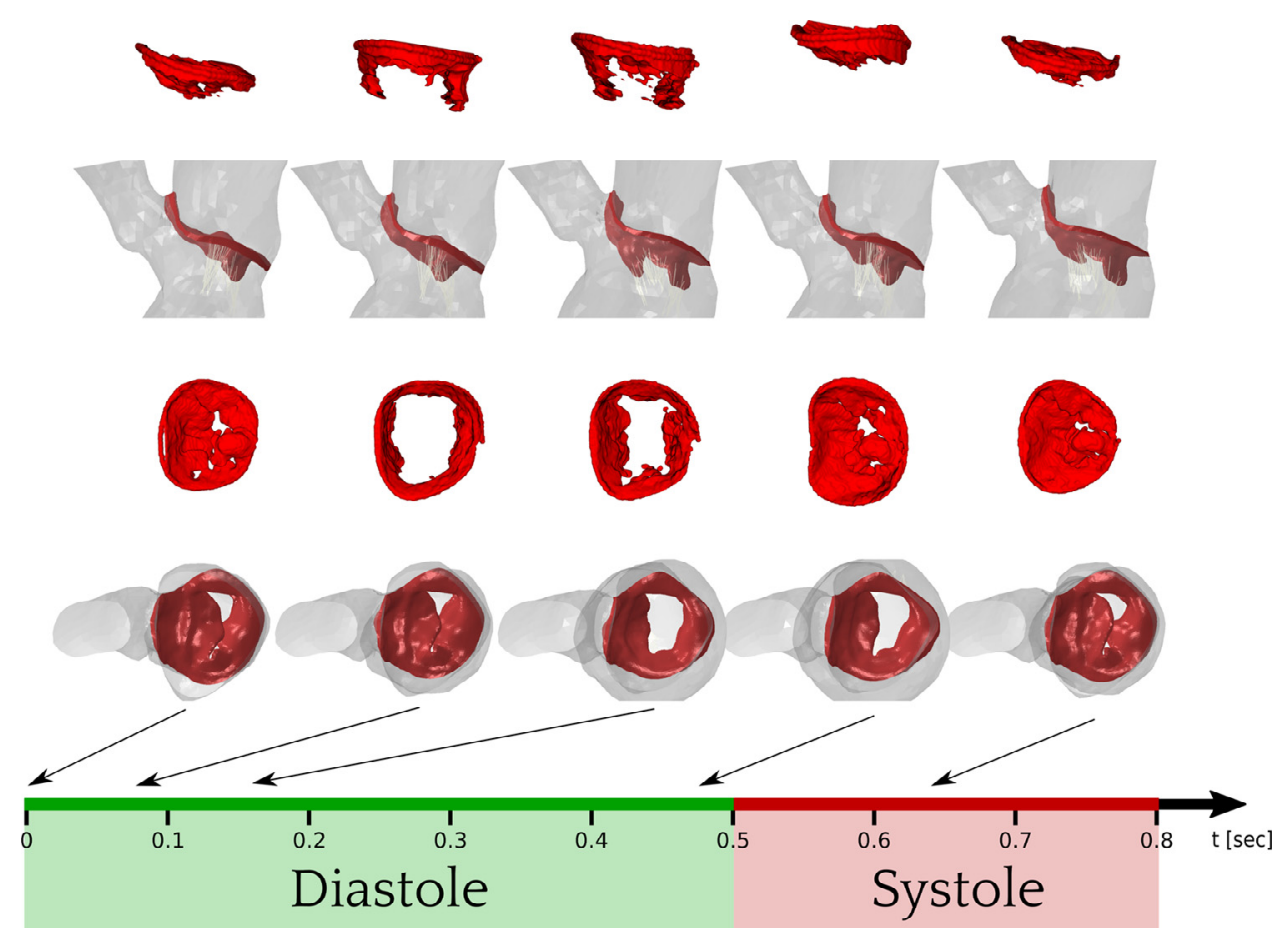

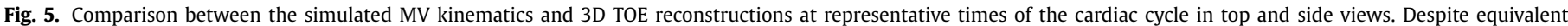

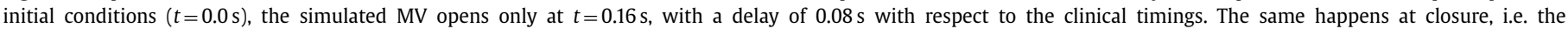
simulated MV closes at $t=0.64 \mathrm{~s}$ while the real one at $t=0.48 \mathrm{~s}$.

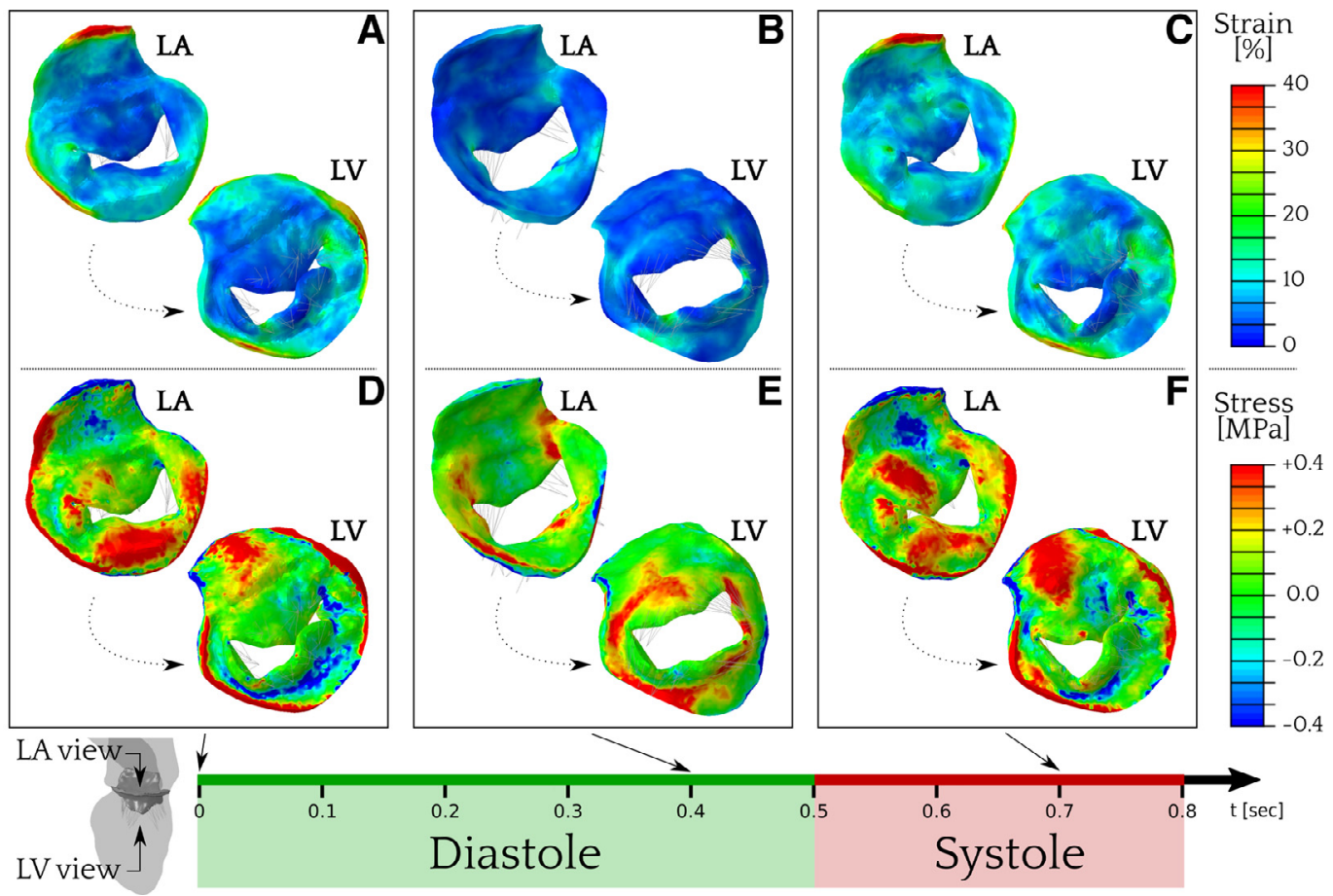

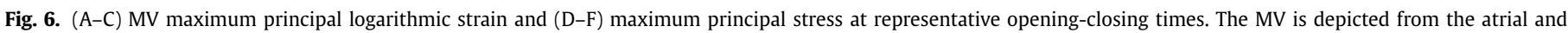

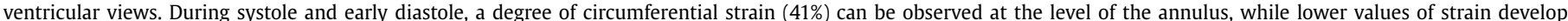

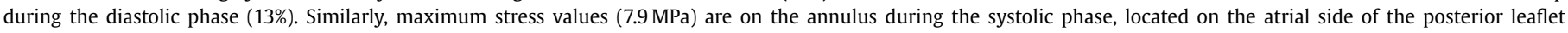

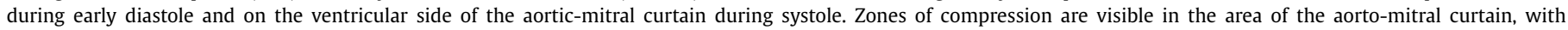
maximum stress values of $11 \mathrm{MPa}$. 


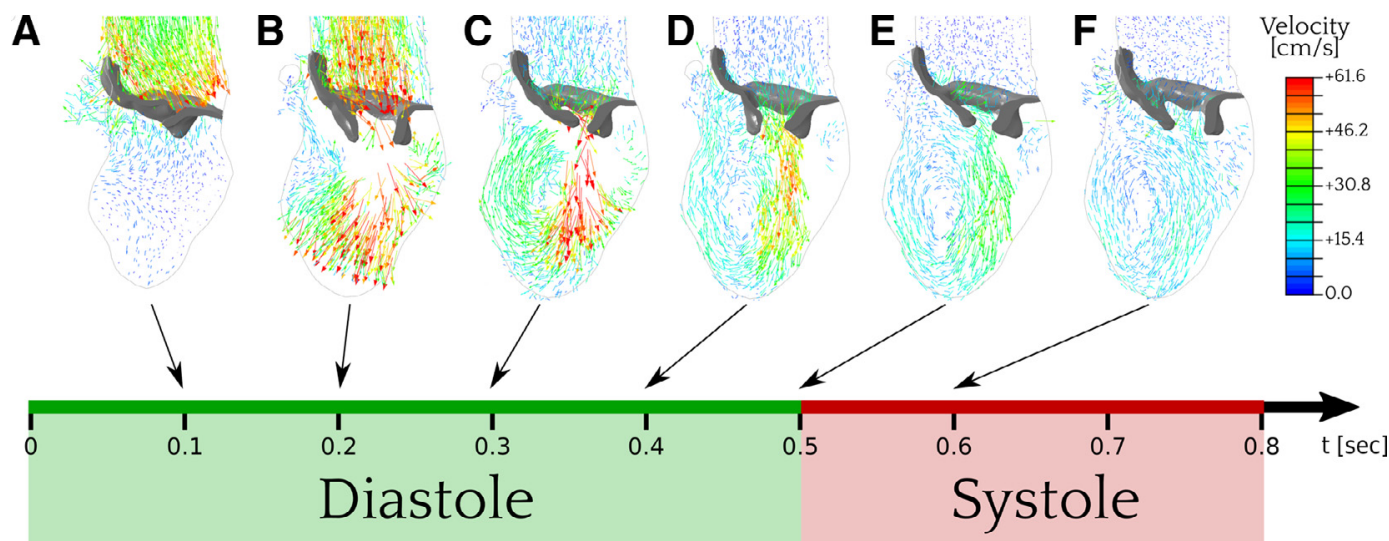

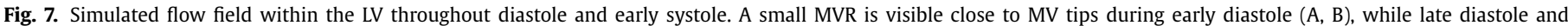
early systole are characterised by a larger clock-wise rotating vortex directed towards the LVOT (C-F).

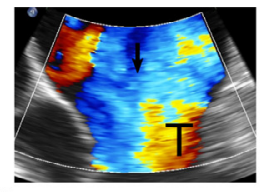

A

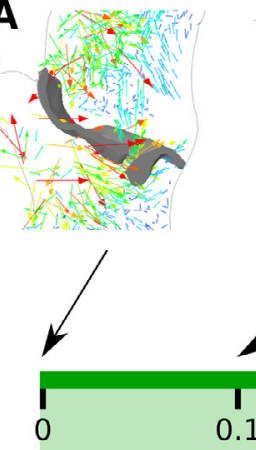

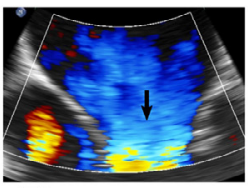

B

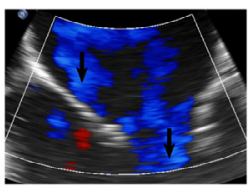

C

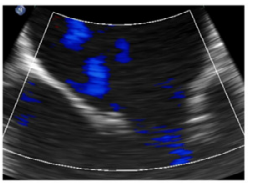

D
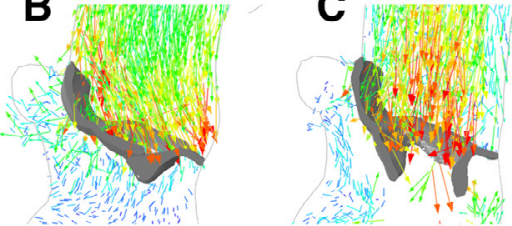

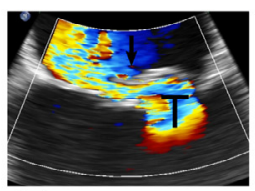

$\mathbf{E}$
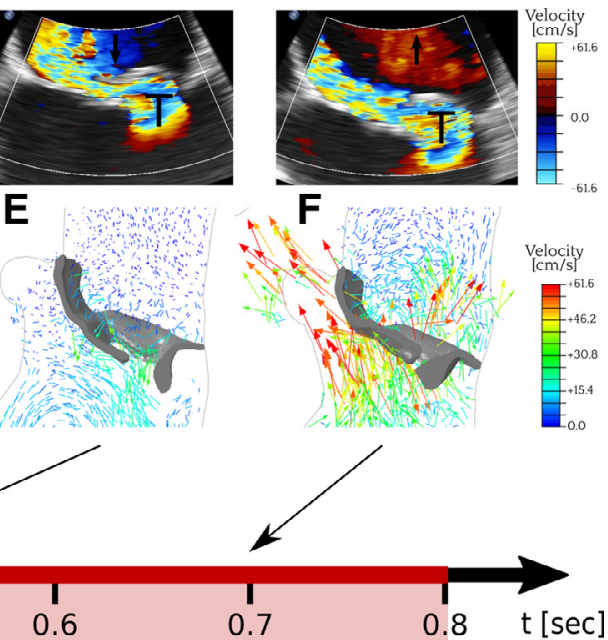

0.2

0.3

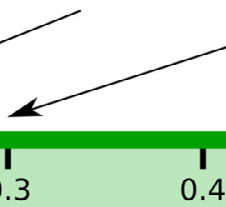

Diastole
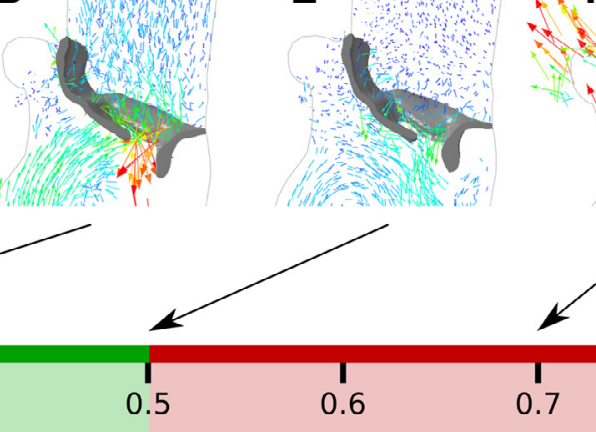

Systole

Fig. 8. Comparison between the simulated flow velocity across the MV and the TOE colour Doppler maps at different times of the cardiac cycle.

tole (C) as a result of annulus and LV contraction. Lower values of strain were registered during the diastolic phase (B) (13\%). Similarly, maximum stress values (7.9 MPa) were reached at the annulus during the systolic phase, located on the atrial side of the posterior leaflet during early diastole (D) and on the ventricular side of the aortic-mitral curtain during systole (F). Compression was seen in the area of the aorto-mitral curtain, with maximum stress values of $11 \mathrm{MPa}(\mathrm{D}, \mathrm{F})$.

In terms of flow assessment, the results of the SPH simulation showed realistic global and local velocity patterns throughout the cardiac cycle. Fig. 7 shows the flow velocity field within the LV during diastole and early systole. Through early diastolic filling (A, B), the development of small vortices in the upper part of the LV due to the roll-up of the shear layer arising from the MV leaflets, i.e. the so-called mitral vortex ring (MVR), was identified. Moreover, a larger, asymmetric, clockwise rotating vortex developed at the centre of the LV towards the end of diastole (C-E), thus facilitating the blood flowing towards the LVOT during systole (F). The rise of these vortex phenomena during ventricular filling has been reported in various computational and clinical studies [36,37].

Moreover, the flow pattern around the MV during systole was compared to the colour Doppler echocardiography maps at different times of the cardiac cycle (Fig. 8). On the colour Doppler images, blue represents areas where the blood is flowing away from the TOE probe, i.e. from LA to LV, while red represents areas where the blood is flowing towards the probe, i.e. from LV to LA. Areas of turbulent flow for which the velocity value cannot be measured by colour Doppler are characterised by a mosaic pattern of colour and indicated with "T" in the pictures. Taking into account the time lag between simulated and actual data already reported above, an overall good correspondence of velocity pattern and direction between simulated and imaging results was found. In particular, the development of an anteriorly directed regurgitant jet can be observed from the simulated frames (Fig. 8-F) as from the Doppler images.

The blood velocity at the level of the MV tip is shown in Fig. 9. Early (E, gravity) and late ( $A$, atrial contraction) filling waves were detected at $t=0.4 \mathrm{~s}$ and $t=0.3 \mathrm{~s}$, with peak mean velocities of $1.33 \mathrm{~m} / \mathrm{s}$ during E-wave and $0.73 \mathrm{~m} / \mathrm{s}$ during A-wave. E/A ratio $=1.82$ and $\mathrm{E}$-wave deceleration time $\mathrm{DT}=90 \mathrm{~ms}$ were in agreement with the patient diagnosis of severe MR. Although transmitral velocity was not measured during TOE, the computed values resulted comparable with pathological ranges in the literature [38-39].

In addition, analysis of the time-dependent flow ejected in the aorta showed a peak velocity during ejection of $1.4 \mathrm{~m} / \mathrm{s}$, in agreement with average values from the literature [38]. By integrating the aortic flow over time, the total volume ejected during systole 


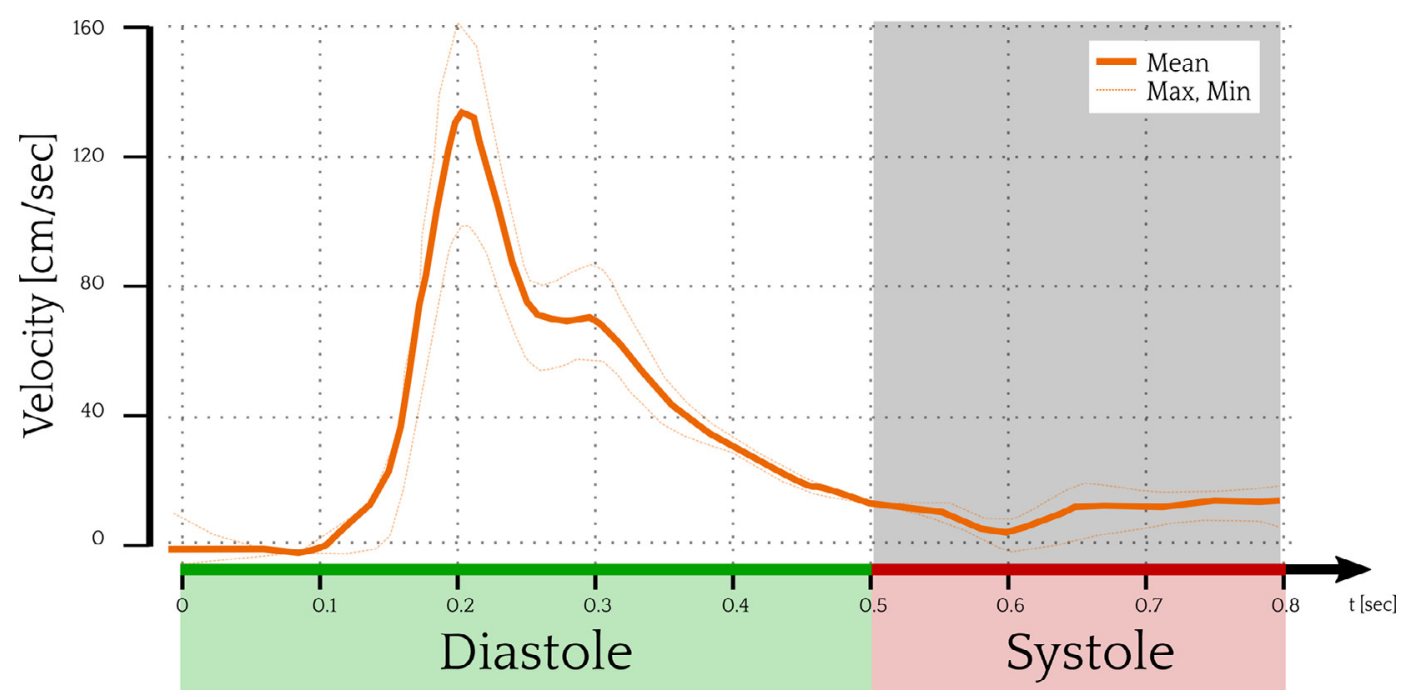

Fig. 9. Blood velocity after the MV (just below the leaflets tip, in open configuration) throughout the cardiac cycle.

Table 1

Numerical values of the available clinical quantities compared with that computed by the simulation. LVEDV- LV end diastolic volume; LVESV- LV end systolic volume; $t_{\text {open }}, t_{\text {close }}-$ MV opening and closing time.

\begin{tabular}{lll}
\hline & Clinical & Simulation \\
\hline LVEDV & $307 \mathrm{ml}$ & $322 \mathrm{ml}$ \\
LVESV & $132 \mathrm{ml}$ & $163 \mathrm{ml}$ \\
$\boldsymbol{t}_{\text {open }}$ & $0.08 \mathrm{~s}$ & $0.16 \mathrm{~s}$ \\
$\boldsymbol{t}_{\text {close }}$ & $0.48 \mathrm{~s}$ & 0.64 \\
MV regurgitant volume & $60-70 \mathrm{ml}$ & $60 \mathrm{ml}$ \\
\hline
\end{tabular}

was estimated at $99 \mathrm{ml}$. By subtracting the latter to the LV SV, the MV regurgitant volume was estimated to be $60 \mathrm{ml}$, corresponding to a regurgitant fraction of $38 \%$ and in agreement with CMR findings (Table 1).

\section{Discussion}

In this study, we have proposed the implementation of a patient-specific model of MV regurgitation, which takes into account both anatomical and functional characteristics through a novel workflow combining multi-modality imaging data and an FSI numerical approach. The model proved to mimic reliably and accurately this case of MV regurgitation, opening the possibility of implementing such modelling for the decision-making process and in-silico device testing.

Patient-specific models of LH and MV were derived from different source clinical data, integrated through a reliable and fully automatic image processing method: CMR is ideal to render both detailed intra-cardiac anatomy and LH dynamics with high spatiotemporal resolution, while the novel 3D TOE enables to capture the complex dynamics of the MV annulus and leaflets with actual thickness. Parallel processing of these large imaging datasets provided automatic generation of both geometry (i.e. anatomical model) and boundary conditions (i.e. LH displacement) with none to minimal user interaction, thus dramatically decreasing processing time and improving accuracy by overcoming user-dependent errors. The generated $\mathrm{LH}$ and MV model was found indeed to be consistent with the clinical observations in terms of ventricle volumes and dynamics, as demonstrated both visually and numerically. The largest difference between clinically measured and automatically reconstructed ventricular volumes was at end systole. This may be due to registration errors between the actual synthetic frame and the $\mathrm{WH}$ image, largest at the end systolic frame, but also to the potential underestimation of the clinical methods of volume calculation based on slice addition, that may not account for the apex slice or for some partial volume at the valve plane.

The SPH method was successfully implemented to simulate the blood flow and its interaction with the MV and cardiac structures. The commercial code allowed a full integration of SPH parts within an FE model, i.e. the fluid particles were automatically generated at the beginning of the simulation and the interaction between the fluid and the solid was handled with a general contact algorithm; no exceptions had to be implemented to guarantee leaflet coaptation, numerically handled as in conventional structural simulations. Such FSI approach was particularly suitable to replicate not only the fluid-dynamics of such a complex apparatus but also the MV kinematics and geometry throughout a full cardiac cycle. As a result, our simulation was able to show local flow patterns within the LV throughout the cardiac cycle, i.e. the development of MVR during early diastole and of a bigger anti-clockwise vortex during late diastole and early systole. This is known to develop as a consequence of the MV eccentricity and in order to facilitate ejection of blood into the aorta, as recent studies investigating intracardiac haemodynamics have demonstrated [40].

The accuracy of the simulation results was evaluated by comparison with medical images. Blood velocity around the MV was in satisfactory agreement with available colour Doppler values. The simulated flow velocity near the MV tips and in the aorta reflected values in literature, giving credit to the reliability of the simulation. Finally, although difficult to quantify directly on the simulation, the regurgitant flow could be indirectly computed from the difference between SV and volume ejected in the aorta providing a regurgitant fraction of $38 \%$, as confirmed by the CMR report and the patient diagnosis of severe MV regurgitation.

Computer simulations can provide additional important information on the patient-specific condition that would be otherwise unachievable with non-invasive in-vivo measurements or through conventional imaging. Local stress and strain fields were in fact analyzed on the MV leaflets modelled with actual variable thickness. Such values could be relevant for understanding tissue degeneration under not physiological loading conditions or in studies of tissue-device interaction.

Albeit an overall good accuracy of the patient-specific MV model was achieved, we identified areas for improvement of the modelling workflow and for further validation. 
We can hypothesise that the main cause of the difference between TOE images and simulated results in terms of MV geometry is the lack of precise patient-specific information about the number and position of the chordae tendinae, as it is well known that these have great influence on MV coaptation. As this information is not retrievable from the patient using medical images available in current clinical practice, the geometry and connection of the simulated chordae was based on data from the literature. However, we do not foresee any issue in adapting our model with patient-specific data as soon as this information becomes available. Furthermore, the MV leaflets were simplified as linear elastic isotropic. This choice was justified by the need of containing both model uncertainties and computational time.

Despite available from CMR reconstruction, the PMs were not included explicitly in the LV mesh, but their presence was simulated by means of a kinematic coupling between the chordae tendinae extremities and the LV wall. The rationale of not including them in the presented model is based on both applied boundary conditions and parameters of interests. The applied kinematic conditions fully govern the dynamic of the LV apparatus in our model. Hence, the papillary muscles would have not had an active role. Therefore, we decided to mimic their presence with kinematic constraints in order to reduce the computational costs. We believe that this choice does not radically affect the main magnitudes of interest of our simulations, i.e. fluido-structure response of the mitral valve. This assumption could be validated in future works by comparing our results with those obtained adding the PM structures in the model.

Although the ratio of systole and diastole was correctly reproduced by the second cycle analysed in this simulation, we reported a slight delay in the simulated MV opening-closing times, due to, first, a component related to the particle residual inertia. The particles are not moving at the beginning of the simulation, and they are therefore accelerated throughout the first cardiac cycle, whose role is to "start the system". We can indeed reasonably hypothesise that simulating more than 2 cardiac cycles would help the system to be fully at speed, and therefore could reproduce the real MV dynamics more accurately. On the other hand, the more the cardiac cycles to simulate, the bigger the particle reservoir required, which would furtherly increase the complexity of the model and the computational cost of the simulation. For this reason, we decided to analyze the results at the end of the second cardiac cycle, identified as the one to provide the best trade-off between accuracy and time. Second, although blood is usually modelled as an incompressible fluid, a small degree of compressibility had to be introduced to increase time increment stability and achieve a reasonable computational time, i.e. a speed of sound of $c_{0}=150 \mathrm{~m} / \mathrm{s}$ was imposed instead of $1500 \mathrm{~m} / \mathrm{s}$.

With regards to the validation of the fluid-dynamic parameters, the lack of velocity values from the clinical data above the Doppler Nyquist threshold did not allow to confirm the simulated velocities around the MV regurgitant jet. In forthcoming studies, we aim to thoroughly verify these values against the corresponding continuous Doppler curves, which were not clinically available for this case. Also, as reported by Belohlavek [37], the TOE could be a suitable image modality to capture intraventricular vortex pattern, and could potentially be used in future works for further validation of the flow results. Given the resulting maximum velocity of $0.6 \mathrm{~m} / \mathrm{s}$, hydraulic diameter $0.02 \mathrm{~m}$, density $1060 \mathrm{~kg} / \mathrm{m} 3$ and viscosity $0.003 \mathrm{~Pa}$, a peak velocity Reynolds of 4240 was found, which is borderline between laminar and turbulent flow. Despite that, the flow behaviour was modelled as laminar, due to the current ABAQUS SPH implementation that does not include turbulence models. Such models may become available in the $\mathrm{SPH}$ formulation in the future which may provide more realistic hemodynamics [41]. Therefore the turbulent pattern of the MV regurgitant jet could only be hypothesised.

In conclusion, our study showed the feasibility of combining multiple image modalities and computer simulations to replicate and investigate the LH and MV mechanics in a pathological patient-specific case. In future work, we aim to apply the presented framework to a larger cohort of simulated cases, which will allow an attempt of statistical validation. Being mostly automatic, the entire workflow from images to FSI simulations has the potential for efficient patient-specific in-silico studies, requiring minimal user input at the pre-simulation stage. The capability and reliability of this approach have been tested in this study, and we envision that the developed tool could serve several purposes, i.e. to better understand the mechanical causes underlying patient-specific MV disease; to test different surgical and interventional solutions, therefore supporting the clinicians with insightful information for planning personalised treatments; and finally to provide device companies with an efficient tool to test and optimise their prototypes in realistic models representing the real pathological anatomy and conditions, hence facilitating the device design process.

\section{Declaration of Competing Interest}

None.

\section{Acknowledgements}

The authors would like to thank the British Heart Foundation and Philips (Eindhoven, Netherlands) for the support with the QLab software.

\section{Funding}

This research was supported by EPSRC-funded UCL Centre for Doctoral Training in Medical Imaging (EP/L016478/1 and EP/N02124X/1), British Heart Foundation (PG/17/6/32797), Action Medical Research Training Fellowship (GN2572). This report is independent research from the National Institute for Health Research Biomedical Research Centre Funding Scheme. The views expressed in this publication are those of the authors and not necessarily those of the National Health Service, the National Institute for Health Research or the Department of Health.

\section{Ethical approval}

Ethical approval was obtained for this study (17LO 1080, London Central Research Ethics Committee).

\section{Supplementary materials}

Supplementary material associated with this article can be found, in the online version, at doi:10.1016/j.medengphy.2019.09. 020 .

\section{References}

[1] McCarthy KP, Ring L, Rana BS. Anatomy of the mitral valve: understanding the mitral valve complex in mitral regurgitation. Eur J Echocardiogr 2010;11(10):i3-9.

[2] Di Mauro M, et al. Functional mitral regurgitation: from normal to pathological anatomy of mitral valve. Int J Cardiol 2013;163(3):242-8.

[3] Adams DH, Rosenhek R, Falk V. Degenerative mitral valve regurgitation: best practice revolution. Eur Heart J 2010;31(16):1958-66.

[4] Maisano F, et al. The future of transcatheter mitral valve interventions: competitive or complementary role of repair vs. replacement? Eur Heart J 2015;36(25870204):1651-9. 
[5] Orban M, et al. Established interventions for mitral valve regurgitation. Current evidence. Herz 2015;41(1):19-25.

[6] Mirabel M, et al. What are the characteristics of patients with severe, symptomatic, mitral regurgitation who are denied surgery? Eur Heart J 2007;28(17350971):1358-65 13 .

[7] Vahanian A, et al. Changing demographics of valvular heart disease and impact on surgical and transcatheter valve therapies. Int J Cardiovasc Imaging 2011;27(21347599):1115-22.

[8] Sorajja P, et al. Transcatheter therapy for mitral regurgitation clinical challenges and potential solutions. Circulation 2017;136(4):404-17.

[9] Preston-Maher GL, Torii R, Burriesci G. A technical review of minimally invasive mitral valve replacements. Cardiovasc Eng Technol 2015;6(25984249):174-84.

[10] Patel A, Bapat V. Transcatheter mitral valve replacement: device landscape and early results. EuroIntervention 2017;13(AA):AA31-9.

[11] Espiritu D, et al. Transcatheter mitral valve repair therapies: evolution, status and challenges. Ann Biomed Eng 2017;45(27234818):332-59.

[12] De Vecchi A, et al. Left ventricular outflow obstruction predicts increase in systolic pressure gradients and blood residence time after transcatheter mitral valve replacement. Sci Rep 2018;8(1):15540.

[13] Marom G. Numerical methods for fluid-structure interaction models of aortic valves. Arch Comput Methods Eng 2014;22(4):595-620.

[14] Kunzelman KS, Einstein DR, Cochran RP. Fluid-structure interaction models of the mitral valve: function in normal and pathological states. Philos Trans R Soc B: Biol Sci 2007;362(1484):1393-406.

[15] Lau KD, et al. Mitral valve dynamics in structural and fluid-structure interaction models. Med Eng Phys 2010;32(9):1057-64 Fluid-structure.

[16] Lau KD, et al. Fluid-structure interaction study of the edge-to-edge repair technique on the mitral valve. J Biomech 2011;44(13):2409-17.

[17] Ma X, et al. Image-based fluid-structure interaction model of the human mitral valve. Comput Fluids 2013;71:417-25.

[18] Griffith BE, et al. Simulating the fluid dynamics of natural and prosthetic heart valves using the immersed boundary method. Int J Appl Mech 2009;1(01):137-77.

[19] Gao H, et al. A finite strain nonlinear human mitral valve model with fluid-structure interaction. Nonlinear mv model with FSI. Int J Numer Method Biomed Eng 2014;30(12):1597-613.

[20] Gao H, et al. Fluid-structure interaction model of human mitral valve within left ventricle. In: Functional imaging and modeling of the heart. Springer International Publishing; 2015. p. 330-7.

[21] Gao H, et al. A coupled mitral valve - left ventricle model with fluidstructure interaction. Med. Eng. \& Phys. 2017;47(1):128-36.

[22] Donea J, et al. Arbitrary Lagrangian-Eulerian methods. In: Encyclopedia of computational mechanics; 2017. p. 1-23.

[23] Peskin CS. Numerical analysis of blood flow in the heart. J Comput Phys $1977 ; 25(3): 220-52$
[24] Monaghan JJ. Smoothed particle hydrodynamics and its diverse applications. Annu Rev Fluid Mech 2012;44(1):323-46.

[25] Toma M, et al. Fluid-structure interaction analysis of papillary muscle forces using a comprehensive mitral valve model with 3D chordal structure. Ann Biomed Eng 2015;44(4):942-53.

[26] Toma M, et al. Fluid-structure interaction analysis of ruptured mitral chordae tendineae. Ann Biomed Eng 2017;45(27624659):619-31.

[27] Ed. by Mao W, et al.Tang Dalin, editor Fully-coupled fluid-structure interaction simulation of the aortic and mitral valves in a realistic 3D left ventricle model. PLoS One 2017;12(9):e0184729 Ed. by.

[28] Rueckert D, et al. Nonrigid registration using free-form deformations: application to breast MR images. IEEE Trans Med Imaging 1999;18(8):712-21.

[29] Modat M, et al. Fast free-form deformation using graphics processing units Comput Methods Programs Biomed 2010;98(3):278-84.

[30] Zuluaga MA, et al. Multi-atlas propagation whole heart segmentation from MRI and CTA using a local normalised correlation coefficient criterion. In: Functional imaging and modeling of the heart. Berlin, Heidelberg: Springer; 2013. p. $174-81$.

[31] Durrleman S, et al. Morphometry of anatomical shape complexes with dense deformations and sparse parameters. Neuroimage 2014;101:35-49.

[32] Biffi B, et al. Investigating cardiac motion patterns using synthetic high-resolution 3D cardiovascular magnetic resonance images and statistical shape analysis. Front Pediatr 2017;5(28337429):34.

[33] Kunzelman KS, Cochran RP. Mechanical properties of basal and marginal mitral valve chordae tendineae. ASAIO Trans 1990;36(2252712):M405-8.

[34] Baillargeon B, et al. The living heart project: a robust and integrative simulator for human heart function. Eur J Mech - A/Solids 2014;48:38-47.

[35] Colagrossi A, Landrini M. Numerical simulation of interfacial flows by smoothed particle hydrodynamics. J Comput Phys 2003;191(2):448-75.

[36] Sotiropoulos F, Le TB, Gilmanov A. Fluid mechanics of heart valves and their replacements. Annu Rev Fluid Mech 2016:48(1):259-83.

[37] Belohlavek M. Vortex formation time: an emerging echocardiographic index of left ventricular filling efficiency? Eur Heart J - Cardiovasc Imaging 2012;13(5):367-9.

[38] Zoghbi WA, et al. Recommendations for noninvasive evaluation of native valvular regurgitation. J Am Soc Echocardiogr 2017;30(4):303-71.

[39] Chahal NS, et al. Normative reference values for the tissue doppler imaging parameters of left ventricular function: a population-based study. Eur J Echocardiogr 2009;11(1):51-6.

[40] Govindarajan V, et al. Synergy between diastolic mitral valve function and left ventricular flow aids in valve closure and blood transport during systole. Sci Rep 2018;8(1).

[41] Monaghan JJ, Mériaux CA. An SPH study of driven turbulence near a free surface in a tank under gravity. Eur J Mech - B/Fluids 2018;68:201-10. 